\title{
Lucro y despojo: el juicio de los trabajadores respecto al sistema previsional chileno
}

\author{
Federico García \\ Departamento de Antropología, Universidad de Chile \\ federico.garal@gmail.com
}

\section{Resumen}

El presente estudio busca dar cuenta de las percepciones e impresiones desarrolladas por los usuarios frente al actual sistema chileno de pensiones. Para ello se han considerado datos provenientes de diferente orígen; primero se considera una encuesta electrónica desplegada en la ciudad de Santiago de Chile entre los meses de Abril y Mayo de 2017 con una muestra, bola de nieve, de 30 casos, material audiovisual referente al tema y, por último, una serie de comentarios extraídos desde la plataforma web del vídeo referido junto con otros obtenidos del perfil de la "Coordinadora Nacional de Trabajadores No más AFP" en la red social Facebook. El análisis propuesto es uno de contenido, eminentemente cualitativo, pero que considera también la presencia de datos cuantitativos descriptivos de la muestra. En forma de hipótesis adelantamos que las percepciones e impresiones respecto del sistema de pensiones son primeramente negativas, dada la articulación de tres elementos; la imposición, beneficios imparciales y una nula voluntad política de cambio en representantes y en el Estado en general. Frente a esta realidad se articula una voluntad política de cambio que instituye un límite entre la adhesión y el rechazo-desinterés a la transformación del actual sistema previsional, generandose una oposición entre usuarios y empresas-Estado.

Palabras clave: AFP, jubilación, lucro, percepciones, trabajadores.

\section{Abstract}

The present study seeks to account for the perceptions and impressions developed by the users of the current Chilean pension system. For this purpose data from different origins have been considered; First an electronic survey was deployed in the city of Santiago de Chile between April and May 2017 with a sample, snowball, 30 cases, audiovisual material on the subject and, finally, a series of comments extracted From the web platform of the video referred to together with others obtained from the profile of the National Coordinator of Workers No more AFP in the social network Facebook. The proposed analysis is one of content, eminently qualitative, but also considering the presence of quantitative data descriptive of the sample. In the form of hypotheses, we anticipate that perceptions and impressions regarding the pension system are primarily negative, given the articulation of three elements; The imposition, impartial benefits and a void political will of change in representatives and in the State in general. Faced with this reality is articulated a political will for change that institutes a limit between the adhesion and rejection-disinterest to the transformation of the current pension system, generating an opposition between users and state-companies.

Key words: AFP, retirement, profit, perceptions, workers. 


\section{Introducción}

Hace ya algunos años múltiples sectores de la sociedad civil chilena se han articulado en función de reivindicar una transformación al actual sistema previsional imperante en el país. De esta manera la cuestión de la jubilación ha sido progresivamente procesada como un problema social que afecta a gran parte sino la mayoría de los trabajadores chilenos, por tanto este tema se ha tomado tribuna en el debate público. En este marco general se muestra como relevante aproximarse, desde una perspectiva antropológica, a las percepciones e impresiones de los trabajadores en relación su afiliación con las administradoras de fondos de pensiones y sus expectativas respecto de la vida en la tercera edad o con posterioridad a sancionada la jubilación.

Como aproximación preliminar el conjunto de los resultados expresan percepciones e impresiones notoriamente negativas. Los elementos que acoplados representan las causas de esta situación giran en torno a que el sistema fue instaurado en dictadura a objeto de beneficiar al empresariado en detrimento de los trabajadores del país y, como tercera arista, nos encontramos con una voluntad política, de los representantes de la sociedad civil y del Estado, que rehuye a construir un sistema que beneficie al conjunto total de trabajadores cotizantes en AFP.

\section{Metodología}

En aras del levantamiento de información se trabajó en tres tareas principales; i) la primera de ellas dice relación con la construcción y posterior difusión de la encuesta referida más arriba, ésta fue configurada a través de las herramienta Google formularios, ii) en segundo lugar se procedió a definir un recurso audiovisual referente al tema en conjunto con los comentarios publicados en la plataforma web en que se encontraba y iii) por último, se extrajeron publicaciones y comentarios dispuestos en el perfil de la "Coordinadora nacional No más AFP" de la red social Facebook.

Con posterioridad se procedió al procesamiento de los datos, para ello se recurrió a las herramientas del software NVivo 11 Pro. A través de éste se codificaron las respuestas obtenidas de la encuesta, el contenido del material audiovisual junto con sus comentarios asociados y las publicaciones extraídas de Facebook. Todas las codificaciones fueron correspondientemente asignadas en una serie de categorías que a su vez conformaron un árbol de nodos. Para el análisis de la información obtenida se debió, en primer lugar, ingresar y procesar los datos a través del software de análisis cualitativo Nvivo, extrayendo relaciones entre nodos y atributos. Una vez obtenidas éstas es posible llevar a cabo el análisis cualitativo de contenido, el cual expresa el sustrato del presente artículo que tiene su reflejo gráfico en los recuadros que mostramos en los anexos. 


\section{Resultados y Análisis}

\section{Sobre los casos}

De los 30 casos procesados 22 corresponden al género femenino, 7 al masculino y 1 a otro. 9 de ellos indicaron estar inscritos en la AFP Modelo, 5 en la AFP Capital, 11 en la AFP Habitat, 3 en Cuprum y 2 en ProVida. Del total el $66,6 \%$ ha cotizado irregularmente o con "lagunas" y el $33,3 \%$ lo ha hecho regularmente. Si cruzamos el atributo género con aquél que corresponde a la empresa AFP de afiliación (ver recuadro) obtenemos que tanto el género femenino como el masculino optan de igual forma por la AFP Modelo en primer lugar, seguida luego de la AFP Capital y en el tercer lugar de preferencia para ambos géneros nos encontramos con la AFP Habitat.

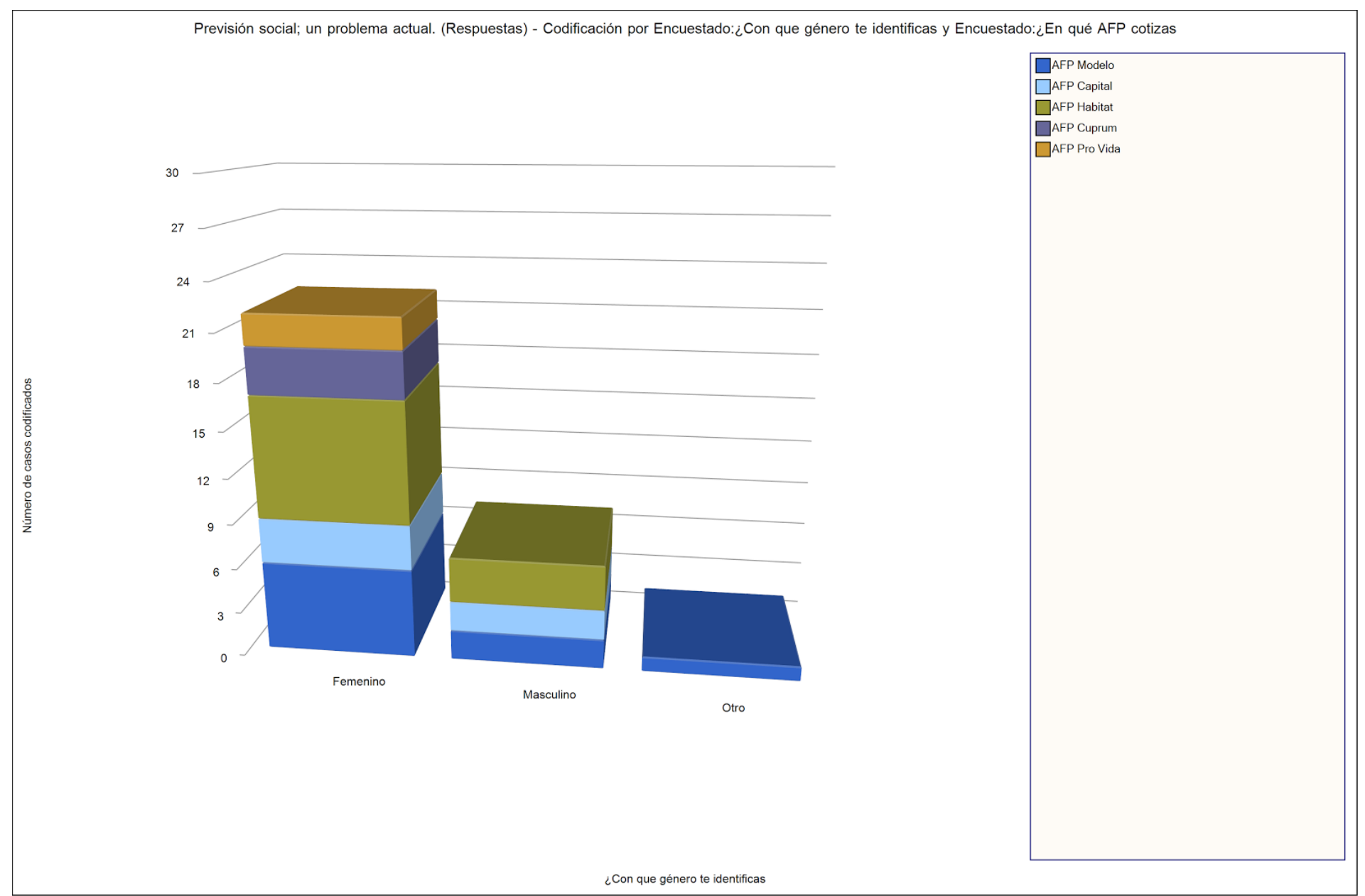

Por su parte advertimos que esta tendencia se mantiene aún introducido el atributo etario y, además, que los más jóvenes (intervalo de 18 a 28 años) prefieren optar por la AFP Modelo mientras que los tres intervalos etarios que le siguen (29 a 39 años, 40 a 50 años y 51 a 61 años) se inclinan por la AFP Habitat. 


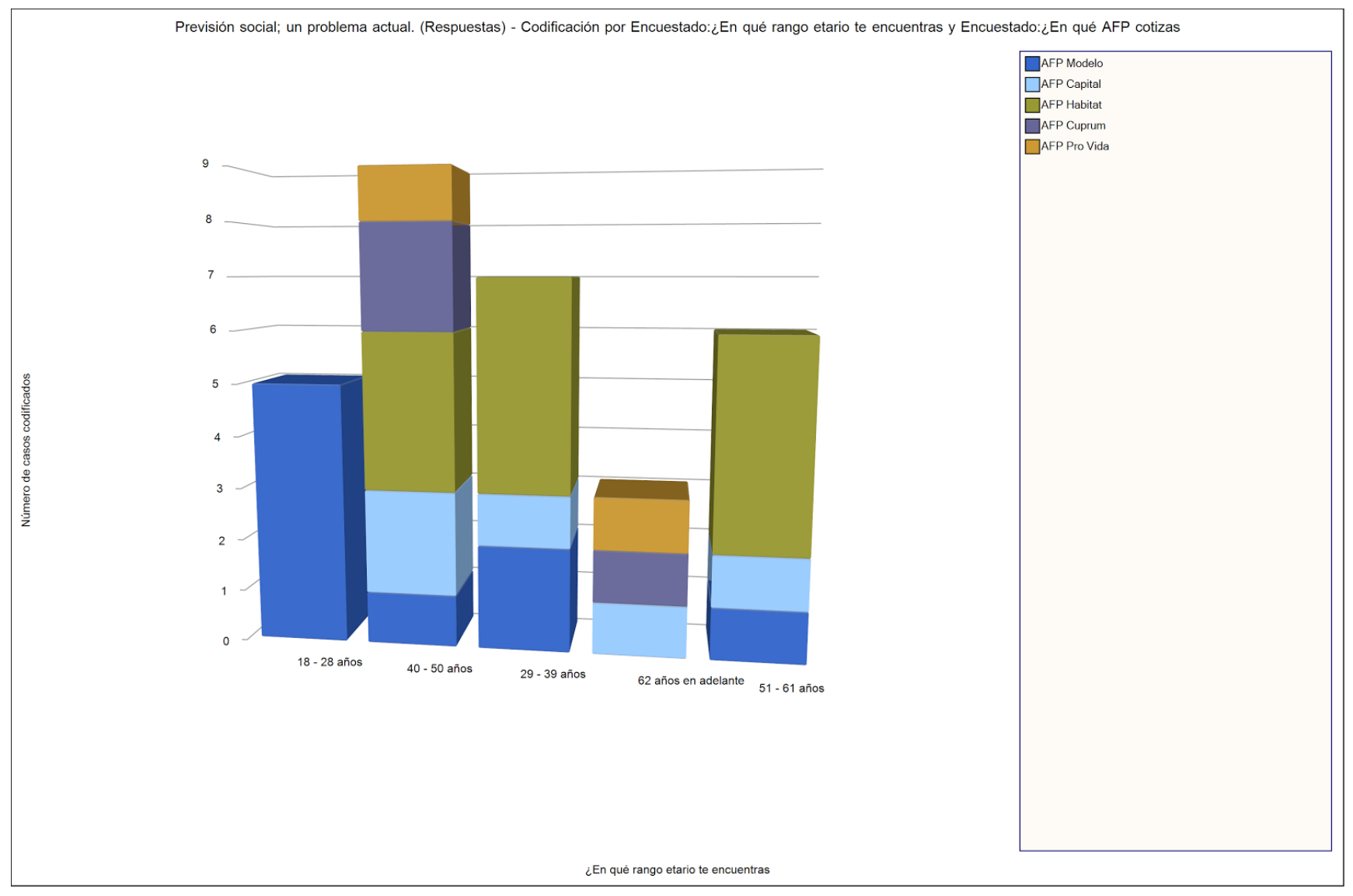

Del total de 30 encuestados el 23,3\% aproxima una pensión futura de entre 100 y 200 mil pesos, el 13,3\% aproxima una jubilación de entre 0 y 100 mil pesos, otro 13,3\% indica que su jubilación se acercará al intervalo de los 200 y 300 mil pesos, el porcentaje restante se divide con un $6,6 \%$ para quienes se jubilarán con una pensión de entre 600 y 700 mil pesos, un 3,3 entre 500 y 600 mil pesos, un $10 \%$ entre 300 y 400 mil pesos y un 3,3\% entre 400 y 500 mil pesos. Como dato relevante tenemos un $26,6 \%$ de los encuestados que señalan indefinición a la hora de aproximar su pensión futura, esta cuestión expresa en buena medida una incertidumbre y/o desinformación de los usuarios frente a los recursos que recibirán luego jubilados.

\section{Historia y Experiencia}

En el eje semántico de la historia y experiencia de cada uno de los casos nos encontramos con una codificación fundamentalmente concentrada en las categorías que hemos denominado "Como condición", la cual hace alusión al imperativo ineludible de afiliación al sistema de AFP toda vez que se esté en empleo activo, y aquella que ha sido nombrada como "Experiencia negativa" que refiere a ocurrencias perjudiciales respecto del desarrollo del sistema de pensiones por AFP. Ambas, en su superposición, indican un nivel de imbricación considerable (ver recuadro 4), esto debido a que gran parte de las experiencias negativas relatadas sobre los diferentes recursos se vinculan con la condición imperativa del sistema de pensiones vigente en Chile. Uno de los casos relata: "El ingreso es automático y muy burocrático. Hasta ahora no se ven beneficios." (Encuestado 20), un segundo caso 
plantea; "Otro punto es el hecho de que no existe alternativa para los trabajadores, todos estamos obligados a cotizar." (Encuestado 24), vemos aquí la correspondencia entre una experiencia adversa y la imposición del sistema de pensiones.

Por su parte la comparación del contenido asignado a la categoría "Como condición" respecto de aquél relacionado con "Ignorancia", que indica cuando el ingreso al sistema de pensiones se llevó a cabo bajo la ignorancia del/la encuestada, presentan un alto grado de correspondencia fundamentalmente dado que el establecimiento del sistema de pensiones por AFP vigente en Chile se desarrolló en desconocimiento de los usuarios (Ver recuadro 5), a modo de ejemplo uno de los encuestados comenta respecto a su experiencia de afiliación a una administradora de pensiones; "Nada especial, mi ingreso fue bien en la ignorancia, era algo que había que hacer." (Encuestado 4). En adición a lo anterior la correlación señala que la obligatoriedad de adhesión al sistema abre paso a una mayor ignorancia respecto de sus condiciones y formas de funcionamiento. En el siguiente caso la ignorancia y la imposición se muestran como causa de la frustración del sujeto; "La primera vez que recibí un sueldo no comprendía porqué se me descontaba un porcentaje si en realidad yo no había decidido eso, me explicaron que así era y no podría no cotizar, eso fue muy frustrante." (Encuestado 1).

\section{Balances e impresiones}

Hacia el interior de los balances e impresiones expresados por los/las encuestadas aparece una tendencia hacia el abordaje de contenidos que asocian al sistema de pensiones con el lucro y el despojo -este último puede asumir una naturaleza material así como una simbólica-, es decir, la predisposición del balance es negativa en tanto la cotización obligatoria significa el desarraigo de un capital privado (ver recuadro 6). Así, el argumento principal que explicaría el conjunto de impresiones hostiles para con el sistema de AFP es que éstas se apropian de parte del patrimonio de cada cotizante. Lo anterior lo podemos ver reflejado en en las citas que siguen:

Pienso que es un sistema injusto y creado para que ciertos grupos económicos ganen dinero a costa del fantasma de la vejez y de la indefensión en la que se encuentra la tercera edad en Chile (...) En resumen me parece un sistema pensado para lucrar de forma bestial con nuestros temores y al final tampoco recibimos ni de cerca lo que hemos cotizado. (Encuestado 24)

Hemos dicho que nos encontramos con un balance fundamentalmente negativo que le atribuye la causa de la precariedad en las pensiones al lucro generado por las administradoras de los fondos de pensiones y el empresariado, pero sumado a ello se agrega un tercer término asociado a la voluntad política (ver recuadro 7), tanto del conjunto de la sociedad civil como de sus representantes por generar cambios al actual sistema. En general la información recopilada da cuenta de una voluntad política positiva de la sociedad civil frente a la posibilidad de un cambio al sistema de pensiones, cuestión que se ratifica con el apoyo constante al perfil de Facebook de la Coordinadora Nacional de Trabajadores No Más AFP y a la adhesión a sus actividades y pronunciamientos públicos. Como ejemplo de lo anterior presentamos un par de comentarios realizados en el el perfil referido; 
Necesitamos un pueblo trabajador organizado y dedicado a terminar con el abuso del sistema previsional. (Comentario Facebook)

Estoy de acuerdo, nosotros debemos decidir qué sistema de pensiones tener.Debemos movilizarnos por el o plebiscito que diga no a las AFP'S. (Encuestado 16)

Por su parte el contenido procesado sugirió que la sociedad civil advierte una negativa voluntad política en sus representantes respecto a la posibilidad de gestar un cambio al actual sistema de pensiones. Podemos, preliminarmente, señalar que el diagnóstico que aquí mostramos puede enmarcarse en un alto nivel de desafección política la sociedad para con sus representantes e instituciones de Estado. Esta percepción además instituye una diferencia entre quienes se benefician del lucro y quienes padecen sus consecuencias, erigiéndose los empresarios y políticos como actores que contravienen los intereses de la sociedad civil, el siguiente caso lo expresa así; "Me gustaría que se cambiara la ley, pero a nuestros parlamentarios no les gustaría cambiar la ley a favor nuestro." (Encuestado 3)

\section{Expectativas y proyecciones}

En cuanto a las expectativas y proyecciones para luego efectuada la jubilación, se manifiesta con alta frecuencia una perspectiva negativa. La muestra indica que por lo general las esperanzas de obtener una jubilación digna son sumamente escasas sino nulas. Así, la unidad de sentido a la que hemos llamado "Expectativas negativas" asumen un nivel importante de superposición con aquella que lleva por nombre "Trabajar" (ver recuadro 8), es decir, la proyección predominante es la de continuar trabajando luego de activada la pensión. Lo anterior se explica básicamente porque las expectativas de recibir pensiones que permitan satisfacer las condiciones básicas de existencia son reducidas, de tal forma que continuar laborando se constituye como una necesidad. Además la expectativa negativa al pensar el futuro se asocia directamente con la precariedad y la insuficiencia del actual sistema de pensiones, la siguiente referencia condensa el planteo anterior; "Como la jubilación no alcanza, probablemente siga trabajando como independiente. La jubilación en Chile es pésima y las personas trabajamos toda la vida para recibir una jubilación deplorable." (Encuestado 20)

No podemos dejar de obviar que varias de las expectativas identificadas en la información procesada dicen relación con el despliegue de actividades extralaborales luego de consumada la jubilación (ver recuadro 9), aún cuando la posibilidad de desarrollar este tipo de ocupaciones se presente como un problema en tanto no generan necesariamente retribución monetaria hacia las personas. Cuando las proyecciones se asocian a los intereses personales de cada individuo estos asumen que su eventual desarrollo se limita por la insuficiencia de los montos asociados a las pensiones. Ejemplo de lo anterior son las declaraciones que ahora mostramos;

Luego de jubilar me gustaría vivir tranquila con la gente que quiero, haciendo lo que me gusta, ocupándome de las temáticas de mi barrio, y sin angustiarme por tener que generar lucas. (Encuestado 13) 
Cuando jubile espero tener tiempo para compartir con mi familia, para compartir con mis vecinos y vecinas, sin tener que trabajar luego de jubilarme. (Encuestado 25)

Mis expectativas luego de jubilar son poder disfrutar de mi dinero descansando o viajando, pero como la mayor parte del tiempo he cotizado con el mínimo para no pagar tanto creo que no tendré lo suficiente para sobrevivir ni una semana. (Encuestado 29)

\section{Comentarios finales}

En primer lugar debemos advertir que los resultados aquí expuestos dan cuenta exclusivamente de las tendencias de la muestra más los elementos extraídos de los recursos ya mencionados, por tanto el análisis propuesto, aún cuando pueda dar luces de un fenómeno general en la sociedad civil, no puede extenderse así sin más al universo de cotizantes en el sistema actual de pensiones. De esta forma el estudio exhibido posee una naturaleza de alcance medio y más bien plantea algunos vértices respecto de la percepción general de los usuarios frente al sistema de pensiones por AFP antes que grandes postulados o tesis vinculadas al tema.

En segundo lugar, y como hemos visto, la historia y las experiencias de los usuarios con el sistema de AFP es comprendida por los mismos como una cuestión negativa que atenta contra la apropiación individual del producto (eminentemente dinero) del trabajo propio. En suma a lo anterior los usuarios identifican que este sistema fue impuesto sin el pleno consentimiento, participación e información de la sociedad civil, fenómeno que genera frustración, impotencia y una naturalización del lucro hacia el interior del sistema de pensiones. Además, como la afiliación a las administradoras de pensiones es obligatorio, el sistema se percibe como una negatividad ineludible.

En concomitancia con lo anterior hallamos un diagnóstico negativo respecto del funcionamiento del sistema de pensiones, fundamentalmente porque éste es asociado por los usuarios al lucro y al despojo del patrimonio de cada cotizante. A su vez esta impresión es comprendida como un efecto propio de las lógicas del gran empresariado y del Estado de Chile, así la reproducción del sistema mismo se encontraría garantizada por la casi inexistente voluntad política de los representantes de la sociedad civil y del gobierno para generar transformaciones o reformas. Esta situación ha producido la institución de una frontera entre quienes se ven afectados nocivamente por el actual sistema y quienes se ven favorecidos por el mismo. Hemos dicho además que este fenómeno debe pensarse en consideración de la significativa desafección política de la sociedad chilena para con las instituciones de Estado y sus representantes.

Frente a un presente que no promete una vejez digna y pensiones que efectivamente garanticen el buen vivir de la tercera edad, las expectativas y proyecciones de los usuarios tienden a colisionar con un margen dispuesto por la necesidad del trabajo, es decir, gran parte proyecta continuar trabajando a fin de lograr costear los gastos que permitan una vida parsimoniosa o al menos estable. Esto último genera que las esperanzas de desarrollar actividades extralaborales o relacionadas con los intereses de cada individuo se minimizan de forma cuantiosa. 
Así todo notamos una disposición positiva de los usuarios para propiciar cambios al actual sistema de pensiones, cuestión que se reafirma con la gran adhesión que ha conseguido el Movimiento Nacional de Trabajadores No Más AFP y, porsupuesto, sus demandas también. Esta voluntad política de transformación se erige como otro de los elementos que aportaría en la emergencia de la frontera a la cual hemos hecho referencia más arriba.

\section{Anexos}

1)
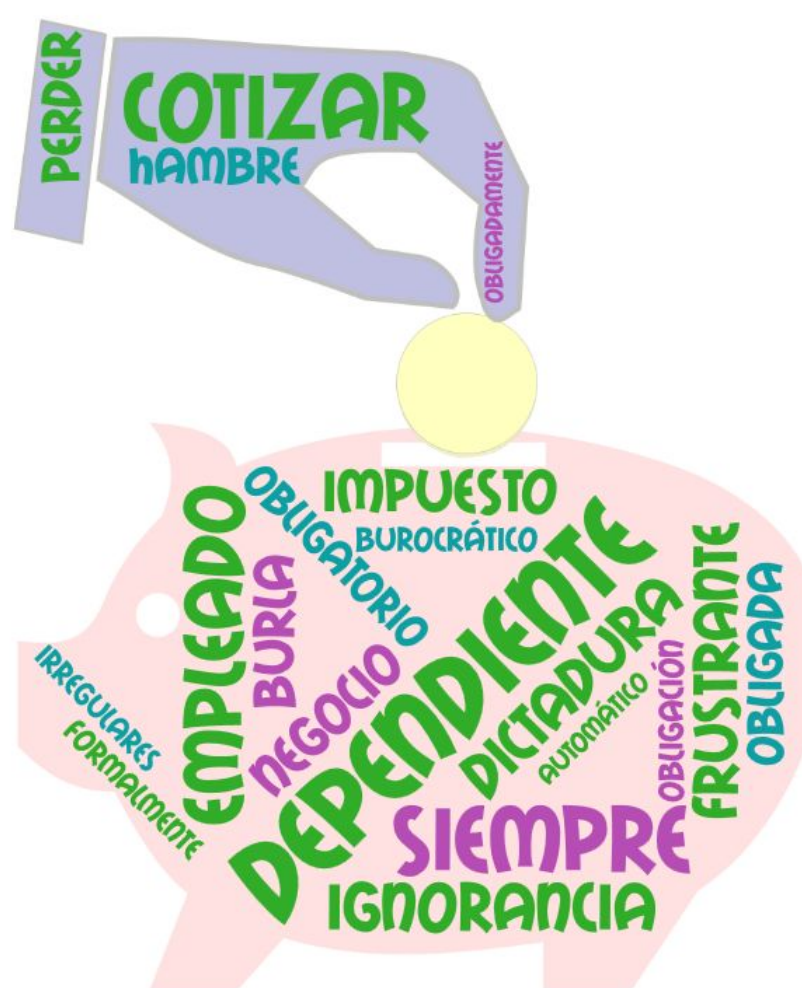

2)

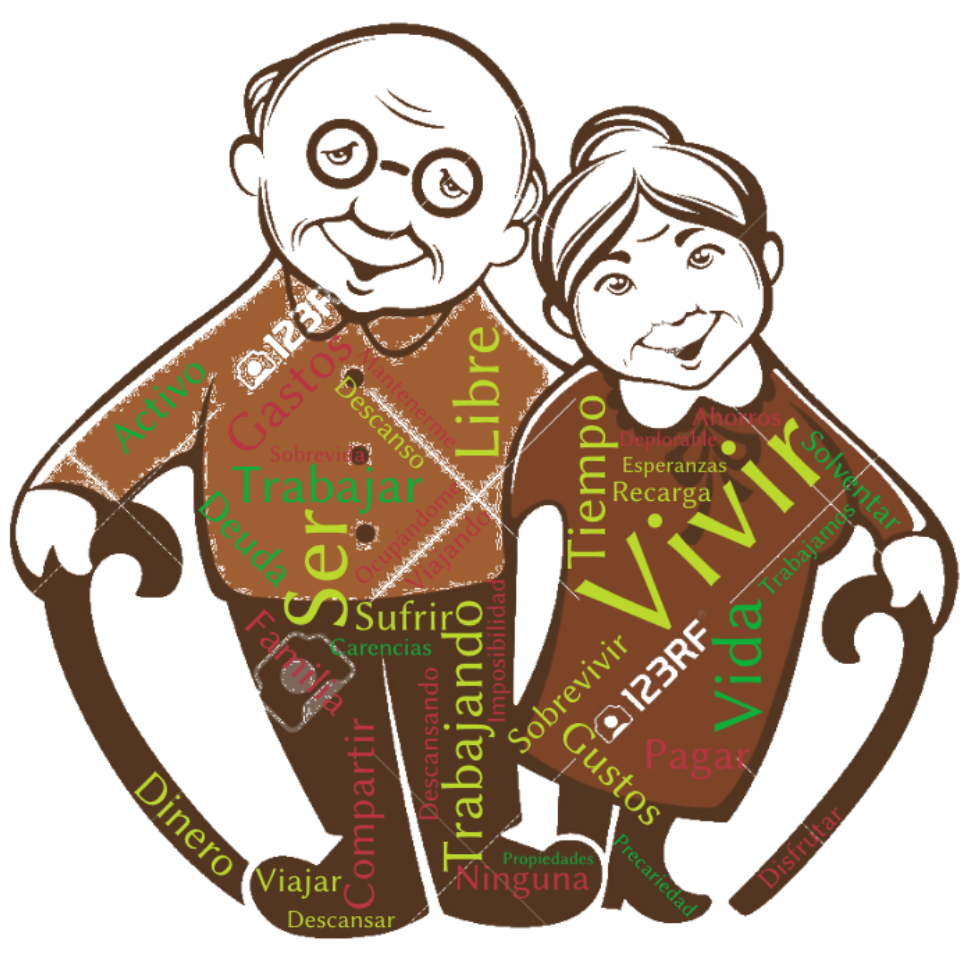


3)

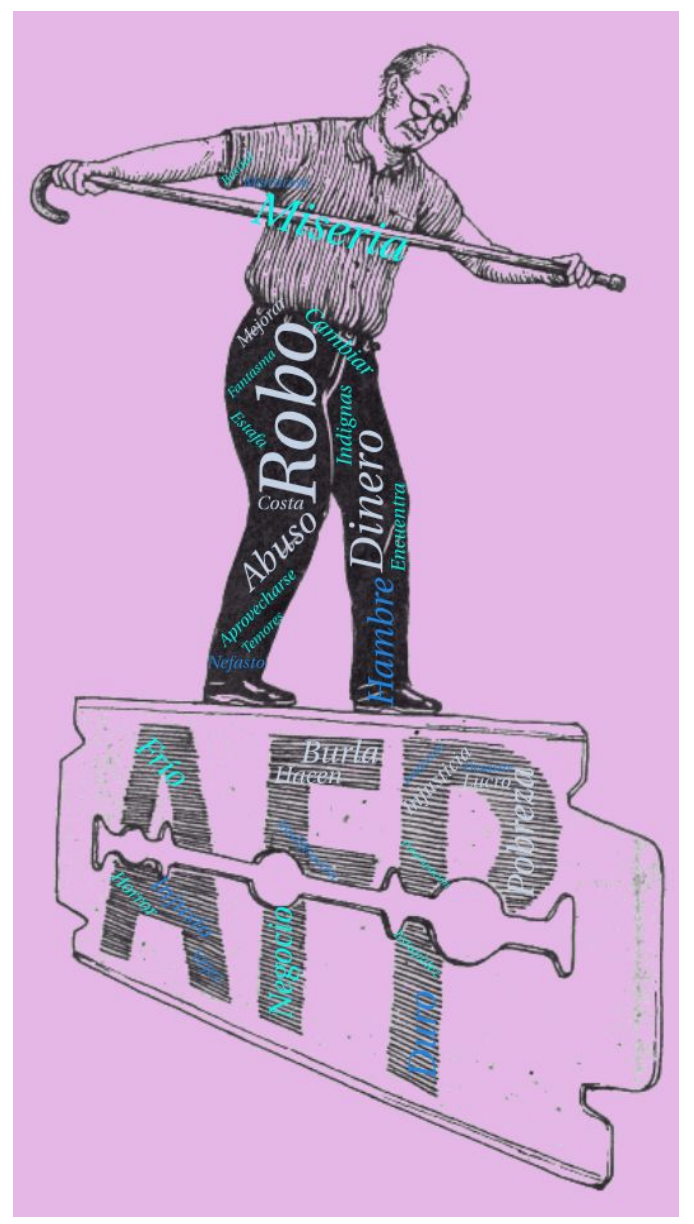

4) 菆目

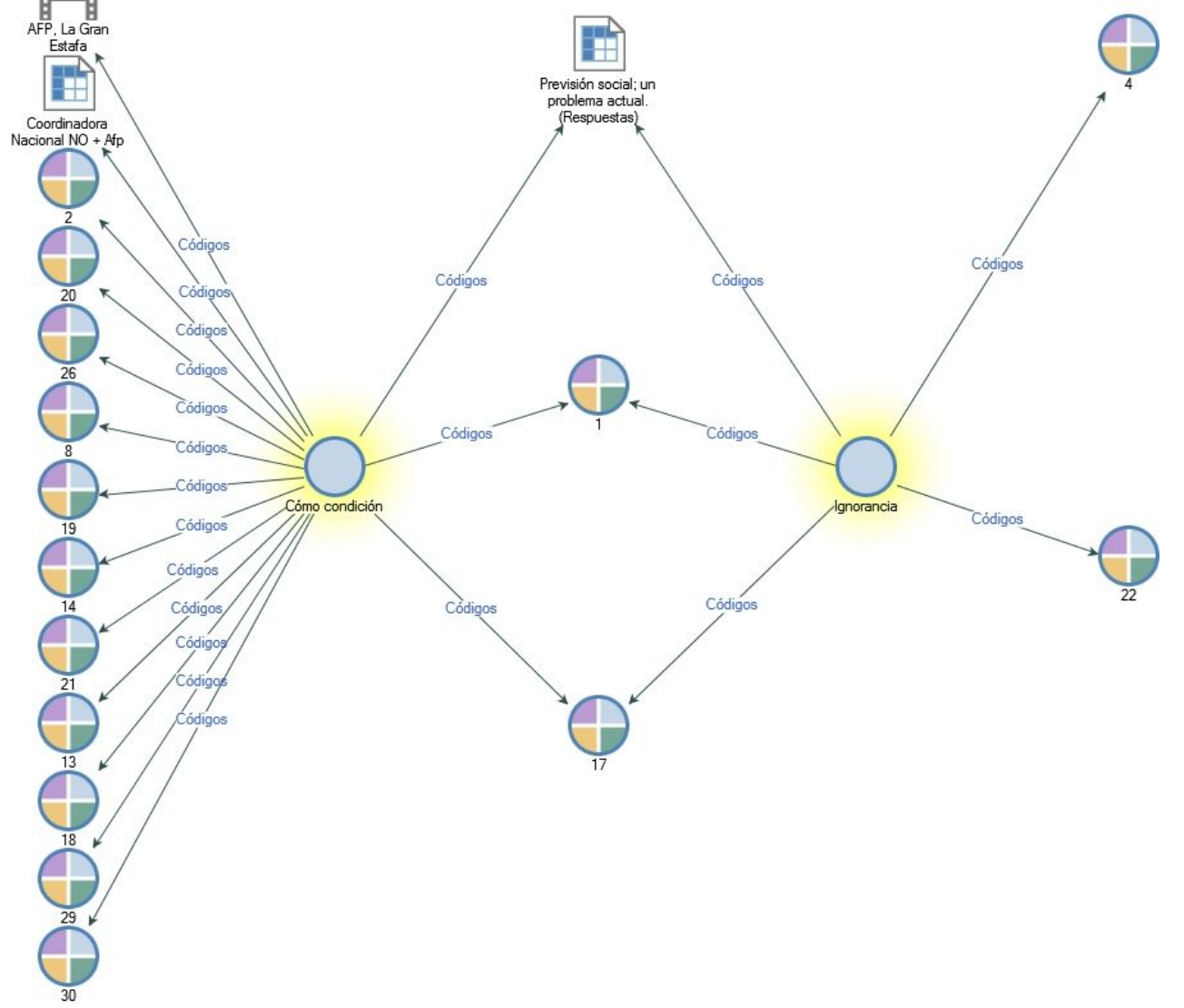


5)

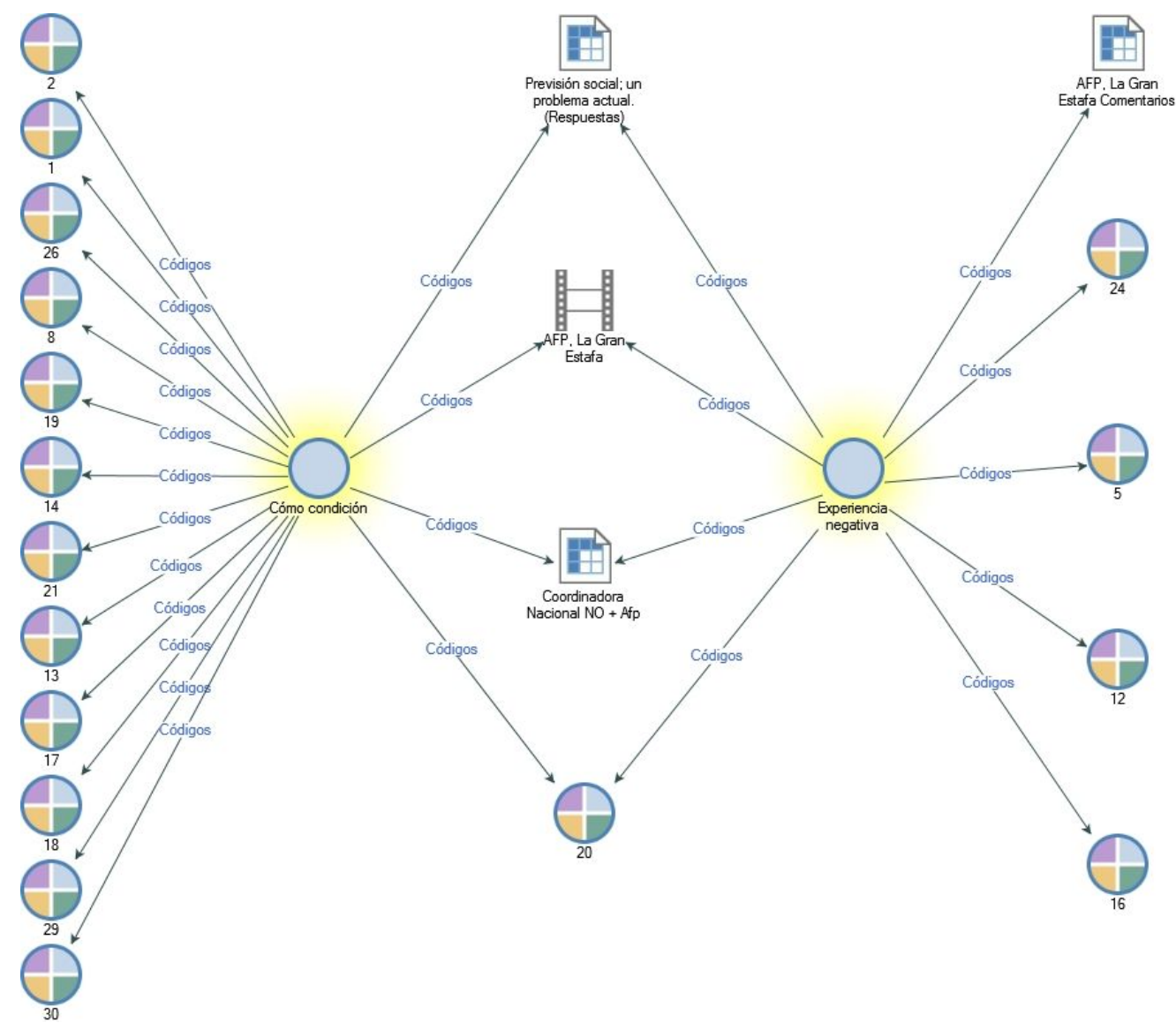

6)

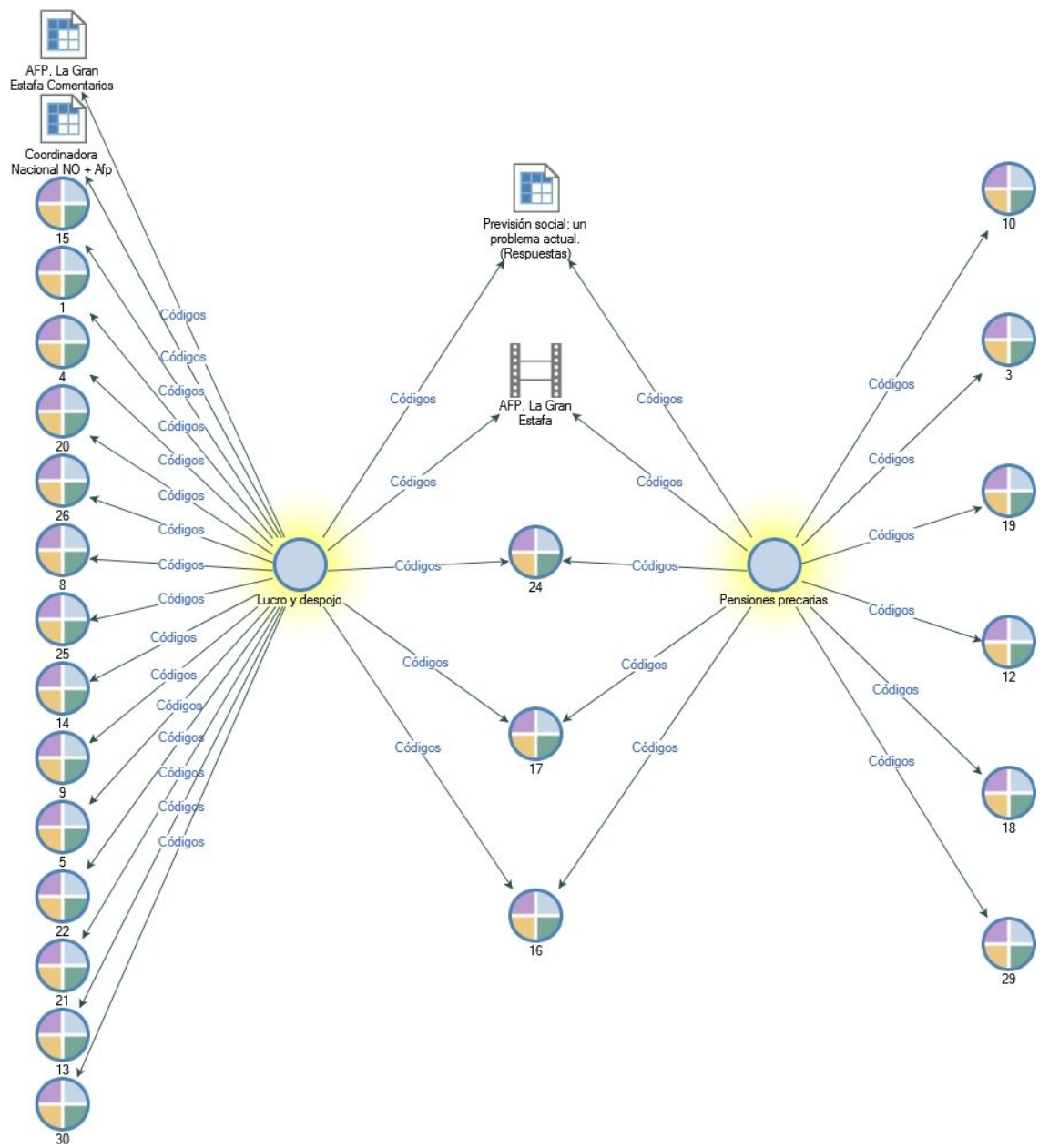


7)



8)

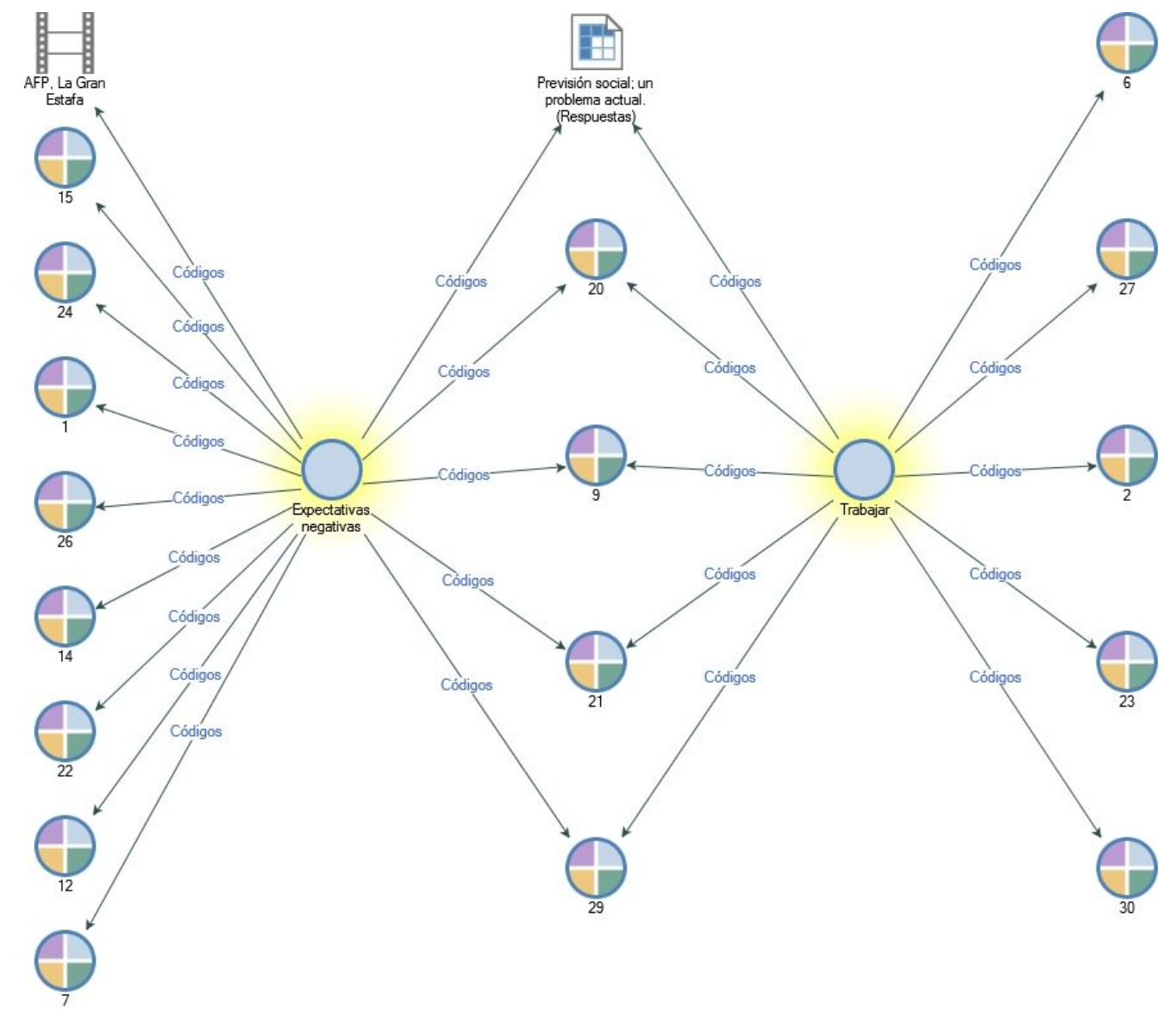


9)

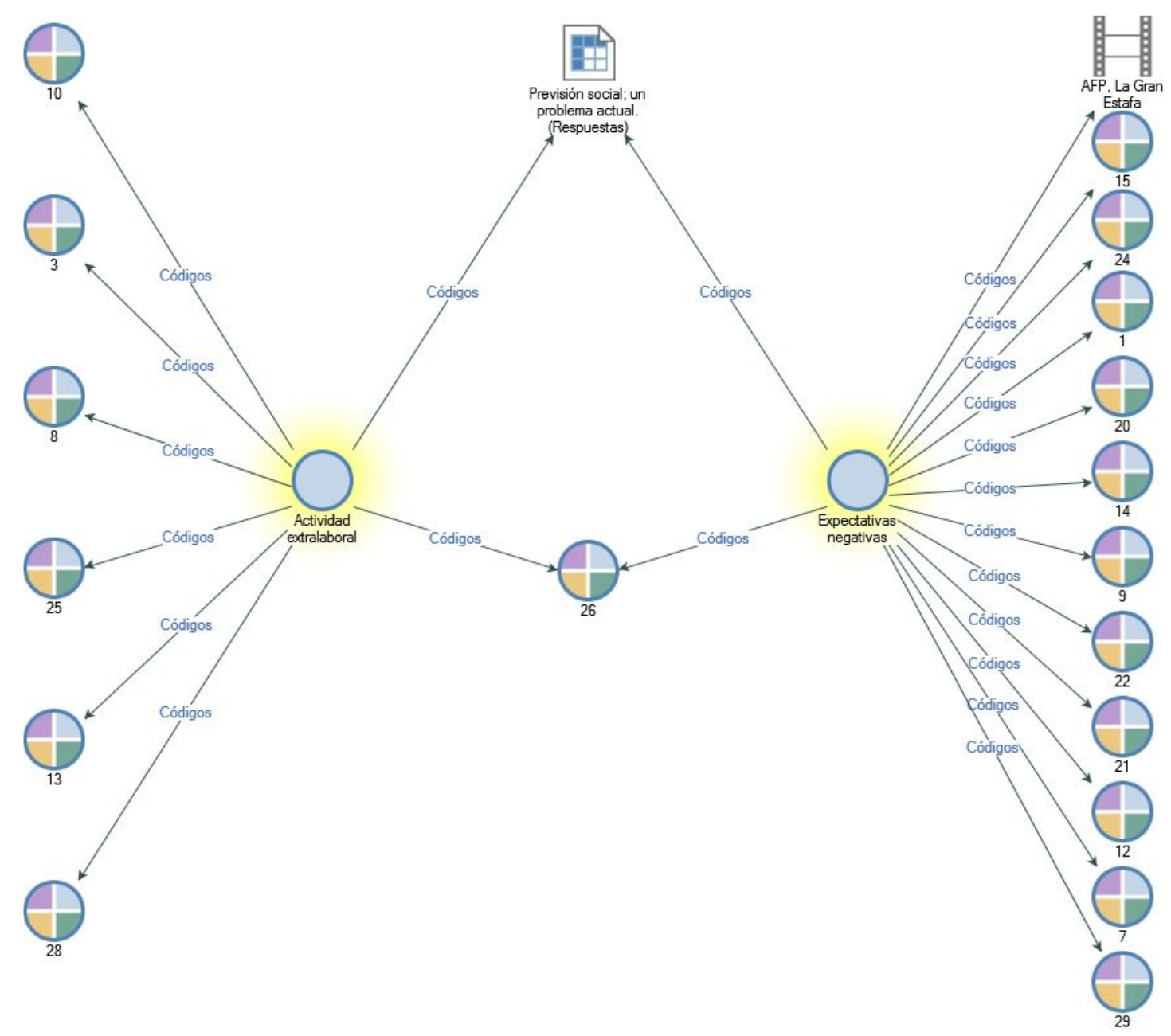

